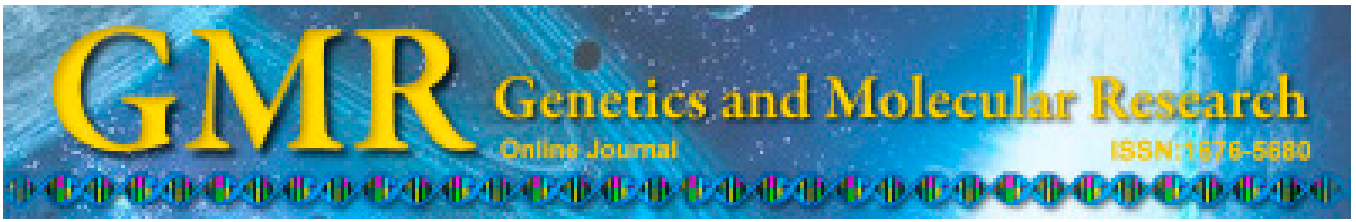

\title{
Effect of leukocyte filtration on the P-selectin expression of apheresis platelets
}

\author{
Z.T. Xie ${ }^{1}$, C. Chen ${ }^{2}$, S.H. Zhang ${ }^{3}$, H.M. Yang ${ }^{1}$ and Z.H. Tao ${ }^{4}$ \\ ${ }^{1}$ Department of Blood Transfusion, \\ The First Affiliated Hospital of Wenzhou Medical University, \\ Wenzhou, China \\ ${ }^{2}$ Department of Laboratory, The Third People's Hospital of Cangnan, \\ Wenzhou, China \\ ${ }^{3}$ Institute of Medical Science, \\ The First Affiliated Hospital of Wenzhou Medical University, \\ Wenzhou, China \\ ${ }^{4}$ Department of Laboratory, \\ The Second Hospital of Zhejiang University School of Medicine, \\ Hanzhou, China \\ Corresponding author: Z.T. Xie \\ E-mail: zuotingxie@163.com
}

Genet. Mol. Res. 14 (2): 5979-5985 (2015)

Received August 25, 2014

Accepted February 5, 2015

Published June 1, 2015

DOI http://dx.doi.org/10.4238/2015.June.1.15

ABSTRACT. The aim of this study was to investigate the effect of leukocyte filtration on the P-selectin (CD62P) surface expression of apheresis platelets during the retention period. Ten bags of apheresis platelets stored for 1 day $(0-24 \mathrm{~h})$ and 10 bags of apheresis platelets stored for 2 days (24-48 h) were used for leukocyte filtration (experimental group). Ten bags of apheresis platelets with the corresponding retention periods but without filtration were used as a negative control (control group). Thereafter, $100 \mu \mathrm{L}$ of platelet suspensions from apheresis platelets with or without leukocyte filtration were sampled before and after leukocyte filtration for the detection of CD62P surface expression by flow cytometry. No statistical difference in the CD62P 
surface expression of apheresis platelets was observed before and after leukocyte filtration ( $\mathrm{P}>0.05)$, neither did the CD62P surface expression exhibit any change among the different retention periods. Leukocyte filtration does not affect the CD62P surface expression of apheresis platelets stored for up to 2 days, which indicates that leukocyte filtration does not damage the activation of apheresis platelets within the retention period.

Key words: Leukocyte filtration; P-selectin; Apheresis platelets

\section{INTRODUCTION}

Allogeneic blood transfusion is an important and indispensable supportive treatment in clinical medicine. However, the presence of blood leukocytes often causes a series of adverse transfusion reactions to occur after platelet transfusion, such as febrile non-hemolytic transfusion reactions, human leukocyte antigen allogeneic immune response, platelet transfusion refractoriness, and philo-leukocyte virus infection (Novotny, 1999; Crescenzi et al., 2012). To reduce the leukocyte-associated adverse transfusion reaction, blood products should be depleted of leukocytes when used clinically. Related research and development work on leukocyte filtration has served as a reference in the preparation of red blood cells, but this process remains in the promotion stage for apheresis platelets. Two main factors limit the use of leukocyte filtration in apheresis platelets: whether leukocyte filtration is necessary for apheresis platelets; and whether the process enhances the activation of platelets in vitro, and thus affects clinical infusion. The necessity of leukocyte filtration for apheresis platelets has been confirmed by several studies and clinical practices (Alhumaidan et al., 2013; Nagura et al., 2013). However, whether the process could result in platelet activation damage is a question that has caused widespread concern and remains unanswered. Laboratory indicators for evaluating the activation of platelets include $\beta$-TG, GPIb (CD42), the platelet aggregation test or GPIIb/IIIa (CD41), soluble P-selectin, platelet surface P-selectin (CD62) (Wasiluk et al., 2013), among which CD62 is the best. Thus, we detected and analyzed the platelet P-selectin (CD62P) surface expression of apheresis platelets with different retention periods using flow cytometry before and after leukocyte filtration to evaluate the effects of leukocyte filtration on platelet activation.

\section{MATERIAL AND METHODS}

\section{Samples}

Twenty bags of apheresis platelets stored for 1 day (0-24 h) and 20 bags of apheresis platelets stored for 2 days (24-48 h) supplied by Wenzhou City Blood Bank were used in this study, among which 10 bags of each retention period were used for leukocyte filtration (experimental group), and the other 10 bags of each retention period without filtration were used as a negative control (control group). Leukocytes in the apheresis platelets were removed at a speed of 13-15 min/bag using platelet-specific leukocyte filters (FTS-PL310, Nanjing Shuangwei Biotechnology Co., Ltd. China) in an International Organization for Standardization Level 6 clean room. Before and after leukocyte filtration, $100 \mu \mathrm{L}$ of platelet suspension was sampled 
from each bag in the experimental group for detection of the expression of CD62P. Thereafter, $100 \mu \mathrm{L}$ of platelet suspension was sampled twice from each bag in the negative control group at the corresponding sampling time and used for detection of CD62P expression.

\section{Flow cytometry}

Platelet suspensions sampled from the experimental and control groups were fixed with $1 \%$ phosphate-buffered paraformaldehyde (Sigma-Aldrich, USA) for $20 \mathrm{~min}$ and then rinsed with phosphate-buffered saline thrice. The suspensions were then centrifuged at 400 $g$ for $5 \mathrm{~min}$. The platelet concentration was adjusted to $5000 / \mu \mathrm{L}$. Thereafter, $50 \mu \mathrm{L}$ of each sample was stained with $5 \mu \mathrm{L}$ anti-CD62P-FITC (BD Biosciences, USA) and $5 \mu \mathrm{L}$ anti-CD41 (BD Biosciences) at room temperature for $30 \mathrm{~min}$ in the dark, and another $50 \mu \mathrm{L}$ of each sample was stained with $5 \mu \mathrm{L}$ mouse IgG1-FITC (BD Biosciences) and $5 \mu \mathrm{L}$ anti-CD41 under the same conditions as the negative control. The platelets were then washed with $2 \mathrm{~mL}$ phosphate-buffered saline and centrifuged at $400 \mathrm{~g}$ for $5 \mathrm{~min}$. Finally, the platelets was resuspended in $300 \mu \mathrm{L} \mathrm{1 \%}$ phosphate-buffered paraformaldehyde and detected on a FACSCalibur flow cytometer (BD Biosciences) using the CellQuest v3.2 software.

\section{Statistical analysis}

Data are reported as means \pm standard deviation (SD) and analyzed using the SPSS v10.0 statistical software (Chicago, IL, USA). A paired $t$-test was used to compare the CD62Ppositive percentage of platelets before and after leukocyte filtration. P values were deemed significant at or below the $5 \%$ level.

\section{RESULTS}

\section{CD62P-positive percentage in control group}

Apheresis platelets stored for 1 day $(0-24 \mathrm{~h})$ or 2 days $(24-48 \mathrm{~h})$ showed no significant difference in terms of CD62P-positive percentage $(\mathrm{P}>0.05$, Figure 1A-F and Table 1).

A-F: The CD62P-positive percentage of apheresis platelets stored for 1 day $(0-24 \mathrm{~h})$ or 2 days (24-48 h). A and D, gated with CD41-PE/FSC; B and E, platelets stained with mouse IgG1 and anti-CD41-PE; $\mathrm{C}$ and F, platelets stained with anti-CD41-PE and anti-CD62P-FITC. A-C, apheresis platelets stored for 1 day ( $0-24 \mathrm{~h})$; D-F, apheresis platelet stored for 2 days (24-48 h).

G-L: The CD62P-positive percentage of apheresis platelets before and after leukocyte filtration. $\mathrm{G}$ and $\mathrm{J}$, gated with CD41-PE/FSC; $\mathrm{H}$ and $\mathrm{K}$, platelets stained with mouse IgG1 and anti-CD41-PE; I and L, platelets stained with anti-CD41-PE and anti-CD62P-FITC. G-I, apheresis platelets before leukocyte filtration; J-L, apheresis platelets after leukocyte filtration; M1, FITC-negative; M2, FITC-positive.

\section{CD62P-positive percentage in experimental group}

The CD62P-positive percentage of the apheresis platelets before leukocyte filtration was not remarkably different from that after leukocyte filtration ( $P>0.05$, Figure $1 \mathrm{G}-\mathrm{L})$, neither was there a large difference between the storage period groups (Table 2). 

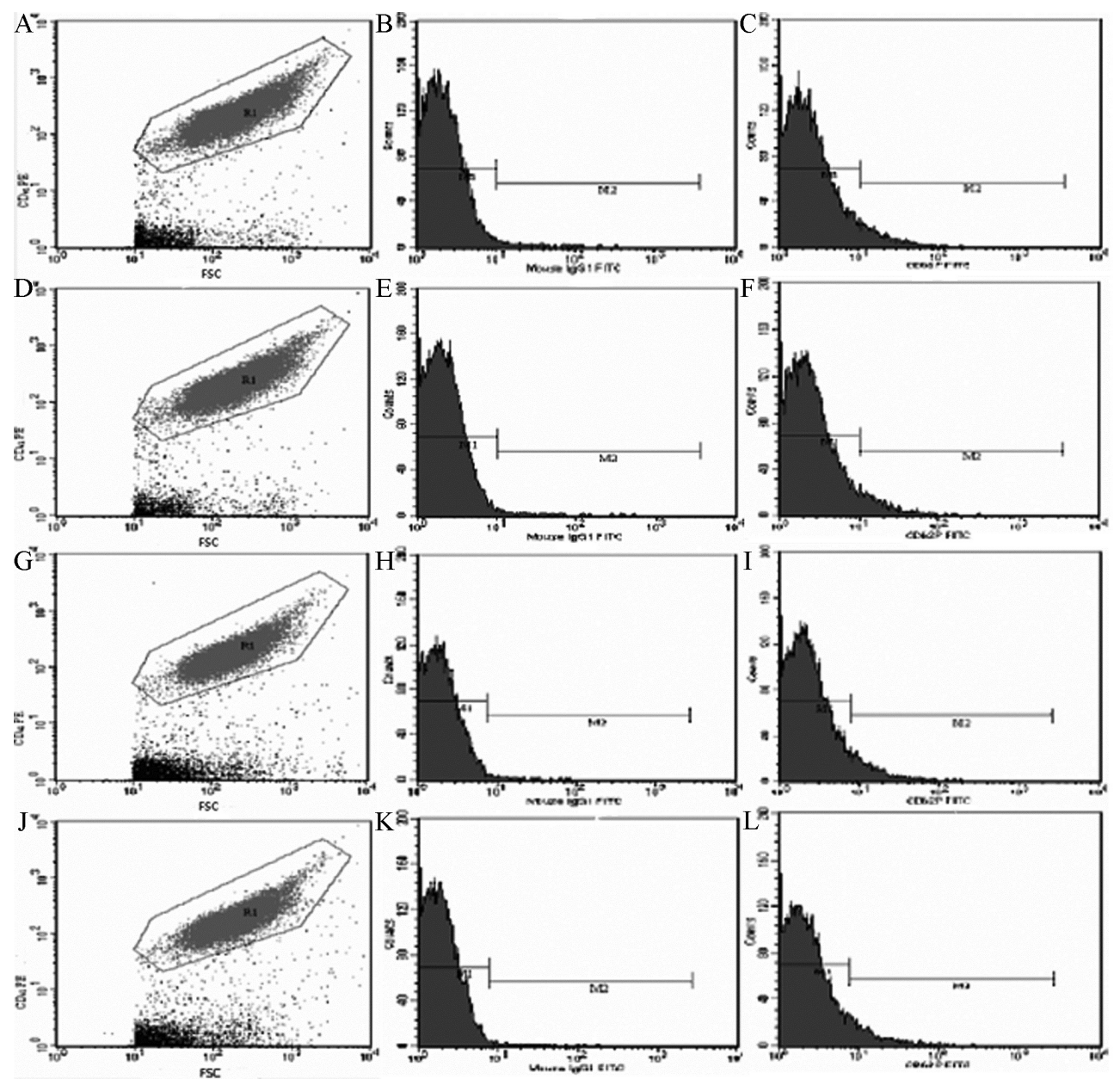

Figure 1. Flow cytometry detection. A.-F. The CD62P-positive percentage of apheresis platelet stored for one day $(0-24$ h) or two days (24-48 h). A. and D. Gated with CD41-PE/FSC; B. and E. platelets stained with mouse IgG1 and anti-CD41-PE; C. and F. platelets stained with anti-CD41-PE and anti-CD62P-FITC. A.-C. Apheresis platelet stored for one day (0-24 h); D.-F. apheresis platelet stored for two day (24-48 h). G.-L. The CD62P-positive percentage of apheresis platelet before and after leukocyte filtration. G and J. Gated with CD41-PE/FSC; H. and K. platelets stained with mouse IgG1 and anti-CD41-PE; I. and L. platelets stained with anti-CD41-PE and antiCD62P-FITC. G.-I. Apheresis platelet before leukocyte filtration; J.-L. apheresis platelet after leukocyte filtration; M1, FITC-negative; M2, FITC-positive.

Table 1. CD62P-positive percentage of apheresis platelet in the negative control.

\begin{tabular}{lccccr}
\hline Groups & Cases & \multicolumn{2}{c}{ CD62P-positive percentage (\%, means \pm SD) } & & P value \\
\cline { 3 - 5 } & & First time & Second time & $t$ value & \\
\hline One day & 10 & $5.74 \pm 2.76$ & $6.32 \pm 2.46$ & 1.460 & $>0.05$ \\
Two days & 10 & $6.06 \pm 2.90$ & $6.97 \pm 2.83$ & 1.579 & $>0.05$ \\
\hline
\end{tabular}




\begin{tabular}{|c|c|c|c|c|c|}
\hline \multirow[t]{2}{*}{ Groups } & \multirow[t]{2}{*}{ Cases } & \multicolumn{3}{|c|}{ CD62P-positive percentage $(\%$, means $\pm \mathrm{SD})$} & \multirow[t]{2}{*}{ Pvalue } \\
\hline & & First time & Second time & $t$ value & \\
\hline One day & 10 & $6.41 \pm 2.85$ & $8.34 \pm 2.55$ & 2.157 & $>0.05$ \\
\hline Two days & 10 & $6.62 \pm 2.11$ & $7.86 \pm 2.71$ & 1.733 & $>0.05$ \\
\hline
\end{tabular}

\section{DISCUSSION}

Leukocyte filtration has been widely used and generalized for whole blood or red blood cell products in many countries, and its safety and clinical value have also been fully affirmed (Eguchi et al., 2007; Sriprawat et al., 2009; Hinojosa and Bryant, 2011; Kim et al., 2013). In some countries, donor blood leukocyte filtration is conducted routinely. Owing to the low leukocyte count remaining in the apheresis platelets (approximately $10^{6}$ to $10^{8}$ ), as well as the consideration that leukocyte filtration may disrupt platelet activation, leukocyte filtration has not been widely used for apheresis platelets. With increasing reports on platelet transfusion refractoriness, the necessity of leukocyte filtration has recently attracted considerable attention (McGettrick et al., 2007; Boodram and Evans, 2008; Singh and Kumar, 2009; Miyaji et al., 2010). Clinical evaluation of the performance of the platelet-specific leukocyte filter merely includes leukocyte removal rate, platelet recoveries, and other indicators (Alaoja et al., 2006; Ott et al., 2006). However, few studies consider whether leukocyte filtration disrupts platelet activation. Considering that platelets are prone to adhesion and aggregation in vitro, the materials and production process of platelet-specific leukocyte filters are key factors in determining the efficiency of leukocyte removal from apheresis platelets. Thus, an erythrocytespecific leukocyte filter cannot be used to remove leukocytes from apheresis platelets. The platelet-specific leukocyte filter used in this study was a flat filter mainly constructed using membranous materials, and it contained medical polyvinyl chloride, acrylonitrile-butadienestyrene plastic, and micro-fiber filters. This type of filter is categorized as a depth filter, the main processing of which involves passing through the molecular sieve and physical/chemical adhesion of the materials. The leukocyte removal rate and platelet recovery rate of this filter can reportedly reach 99.8 and $91.29 \%$, respectively (Guerid et al., 2013).

CD62P, also known as platelet $\alpha$-granule membrane protein-140 (GMP-140), is expressed on the platelet plasma membrane during platelet activation, but not on the resting platelet. CD62P can reportedly transfer rapidly to the platelet surface when the platelet is activated (Ritchie et al., 2000; Vetlesen et al., 2007). Among the surface-activated markers of platelets, CD62P expression shows the most significant difference between activated and non-activated platelets. Thus, CD62P is generally considered the most sensitive and specific marker reflecting platelet activation. Holme et al. (1997), and Dumont and VandenBroeke (2003) reported that a relationship exists between the expression of P-selectin and the life of a platelet after transfusion: the more strongly P-selectin expresses on the platelet surface, the more easily platelets are cleared out after platelet transfusion. CD41, also called platelet membrane glycoprotein IIb/IIIa (GPIIb/IIIa), is stably expressed on both the activated and non-activated platelet surface and can thus be used as a platelet marker. In this study, CD41 ${ }^{+}$ was used to label the platelets, whereas the positive percentage of $\mathrm{CD} 41^{+} \mathrm{CD} 62 \mathrm{P}^{+}$was used to reflect the degree of platelet activation. 
To avoid the influence of non-research factors, a negative control group was set up in this study. No statistical difference was observed between the two sampling times or among the different retention periods, suggesting that sampling time is not a confounding factor that would result in platelet activation. Thus, leukocyte filtration can be regarded as the single factor. Our results showed no significant difference between CD62P expression before and after leukocyte filtration, irrespective of the retention periods. This suggests that filtration has no effect on the CD62P expression of the apheresis platelets regardless of whether they were stored for 1 or 2 days. Thus, we can conclude that leukocyte filtration may not cause activation damage to the apheresis platelets during retention. However, we notably failed to obtain sufficient apheresis platelets with the retention periods of 3,4 , and 5 days (the expiration date for apheresis platelets stored in platelet-specific bags and oscillated at $22^{\circ} \pm 2^{\circ} \mathrm{C}$ ) to analyze CD62P expression. Therefore, whether leukocyte filtration can promote platelet activation over such retention periods remains unclear.

Collectively, leukocyte filtration by a platelet-specific leukocyte filter does not promote the platelet activation of apheresis platelets stored for up to 2 days. Thus, leukocyte filtration can be recommended for use with apheresis platelets for such a retention period to prevent the occurrence of leukocyte-associated adverse reactions after platelet transfusion.

\section{Conflicts of interest}

The authors declare no conflict of interest.

\section{REFERENCES}

Alaoja H, Niemelä E, Anttila V, Dahlbacka S, et al. (2006). Leukocyte filtration to decrease the number of adherent leukocytes in the cerebral microcirculation after a period of deep hypothermic circulatory arrest. J. Thorac. Cardiovasc. Surg. 132: 1339-1347.

Alhumaidan HS, Cheves TA, Holme S and Sweeney JD (2013). The effect of filtration on residual levels of coagulation factors in plasma. Am. J. Clin. Pathol. 139: 110-116.

Boodram S and Evans E (2008). Use of leukocyte-depleting filters during cardiac surgery with cardiopulmonary bypass: a review. J. Extra Corpor. Technol. 40: 27-42.

Crescenzi G, Torracca L, Capestro F, Matteucci ML, et al. (2012). Allogenic blood transfusion in cardiac surgery. J. Card. Surg. 27: 594-599.

Dumont LJ and VandenBroeke T (2003). Seven-day storage of apheresis platelets: report of an in vitro study. Transfusion 43: $143-150$

Eguchi K, Saito K, Kondo M, Hidaka T, et al. (2007). Enhanced effect of high-dose leukocytapheresis using a large filter in rheumatoid arthritis. Mod. Rheumatol. 17: 481-485.

Guerid S, Darwiche SE, Berger MM, Applegate LA, et al. (2013). Autologous keratinocyte suspension in platelet concentrate accelerates and enhances wound healing - a prospective randomized clinical trial on skin graft donor sites: platelet concentrate and keratinocytes on donor sites. Fibrogenesis Tissue Repair 6: 8.

Hinojosa R and Bryant BJ (2011). WBC reduction filtration efficacy performed at varying time intervals post-collection. Transfusion 51: 2758-2760.

Holme S, Sweeney JD, Sawyer S and Elfath MD (1997). The expression of p-selectin during collection, processing, and storage of platelet concentrates: relationship to loss of in vivo viability. Transfusion 37: 12-17.

Kim JM, Kim GS, Joh JW, Suh KS, et al. (2013). Long-term results for living donor liver transplant recipients with hepatocellular carcinoma using intraoperative blood salvage with leukocyte depletion filter. Transpl. Int. 26: 84-89.

McGettrick HM, Filer A, Rainger GE, Buckley CD, et al. (2007). Modulation of endothelial responses by the stromal microenvironment: effects on leucocyte recruitment. Biochem. Soc. Trans. 35: 1161-1162.

Miyaji K, Miyamoto T, Kohira S, Itatani K, et al. (2010). The effectiveness of prestorage leukocyte-reduced red blood cell transfusion on perioperative inflammatory response with a miniaturized biocompatible bypass system. J. Thorac. Cardiovasc. Surg. 139: 1561-1567. 
Nagura Y, Tsuno NH, Tanaka M, Matsuhashi M, et al. (2013). The effect of pre-storage whole-blood leukocyte reduction on cytokines/chemokines levels in autologous CPDA-1 whole blood. Transfus. Apher. Sci. 49: 223-230.

Novotny VM (1999). Prevention and management of platelet transfusion refractoriness. Vox. Sang. 76: 1-13.

Ott R, Bussenius-Kammerer M, Reck T, Hering C, et al. (2006). Does the inactivation of leukocytes in blood transfusions during and following liver transplantation by gamma-irradiation have an impact on rejection and infection rate? Med. Sci. Monit. 12: CR514-CR520.

Ritchie JL, Alexander HD and Rea IM (2000). Flow cytometry analysis of platelet P-selectin expression in whole blood methodological considerations. Clin. Lab. Haematol. 22: 359-363.

Singh S and Kumar A (2009). Leukocyte depletion for safe blood transfusion. Biotechnol. J. 4: 1140-1151.

Sriprawat K, Kaewpongsri S, Suwanarusk R, Leimanis ML, et al. (2009). Effective and cheap removal of leukocytes and platelets from Plasmodium vivax infected blood. Malar. J. 8: 115.

Vetlesen A, Mirlashari MR, Ezligini F and Kjeldsen-Kragh J (2007). Evaluation of platelet activation and cytokine release during storage of platelet concentrates processed from buffy coats either manually or by the automated OrbiSac system. Transfusion 47: 126-132.

Wasiluk A, Kemona H, Mantur M, Polewko A, et al. (2013). Expression of P-selectin (CD62P) on platelets after thrombin and ADP in hypotrophic and healthy, full-term newborns. J. Matern. Fetal. Neonatal. Med. 26: 1321-1324. 